\title{
The symmetry energy at suprasaturation density and the ASY- EOS experiment at GSI
}

E. De Filippo ${ }^{1, a}$, P. Russotto ${ }^{1}$, L. Acosta ${ }^{2,3}$, M. Adamczyk ${ }^{4}$, A. Al-Ajlan ${ }^{5}$, M. Al-Garawi ${ }^{6}$, S. AlHomaidhi $^{5}$, F. Amorini ${ }^{2}$, L. Auditore ${ }^{7,8}$, T. Aumann ${ }^{9,10}$, Y. Ayyad ${ }^{11}$, Z. Basrak ${ }^{12}$, J. Benlliure ${ }^{11}$, M. Boisjoli ${ }^{13}$, K. Boretzky ${ }^{10}$, J. Brzychczyk ${ }^{4}$, A. Budzanowski ${ }^{14}$, C. Caesar ${ }^{9}$, G. Cardella ${ }^{1}$, P. Cammarata $^{15}$, Z. Chajecki ${ }^{16,17}$, M. Chartier ${ }^{18}$, A. Chbihi ${ }^{10}$, M. Colonna ${ }^{2}$, M.D. Cozma ${ }^{19}$, B. Czech $^{14}$, M. Di Toro ${ }^{2,20}$, M. Famiano ${ }^{21}$, S. Gannon ${ }^{18}$, I. Gašparić ${ }^{9,12}$, L. Grassi' ${ }^{12}$, C. Guazzoni ${ }^{22,23}$, P. Guazzoni ${ }^{22,24}$, M. Heil ${ }^{10}$, L. Heilborn ${ }^{15}$, R. Introzzi ${ }^{25}$, T. Isobe ${ }^{26}, \mathrm{~K}_{\text {Kezzar }}$, M. Kiš ${ }^{10}$, A. Krasznahorkay ${ }^{27}$, S. Kupny ${ }^{4}$, N. Kurz ${ }^{10}$, E. La Guidara1, G. Lanzalone ${ }^{2,28}$, P. Lasko ${ }^{4}$, A. Le Fèvre ${ }^{10}$, Y. Leifels ${ }^{10}$, R.C. Lemmon ${ }^{29}$, Q.F. Li ${ }^{30}$, I. Lombardo ${ }^{31,32}$, J. Łukasik ${ }^{14}$, W.G. Lynch $^{16}$, P. Marini ${ }^{13,15}$, Z. Matthews ${ }^{18}$, L. May ${ }^{15}$, T. Minniti ${ }^{1}$, M. Mostazo ${ }^{11}$, A. Pagano ${ }^{1}$, E.V. Pagano ${ }^{2,20}$, M. Papa ${ }^{1}$, P. Pawłowski ${ }^{14}$, S. Pirrone ${ }^{1}$, G. Politi ${ }^{1,20}$, F. Porto ${ }^{2,20}$, W. Reviol ${ }^{33}$, F. Riccio 22,23 , F. Rizzo 2,20 , E. Rosato ${ }^{31,32}$, D. Rossi ${ }^{9,10}$, S. Santoro 7,8 , D.G. Sarantites ${ }^{33}$, H. Simon ${ }^{10}$, I. Skwirczynska ${ }^{14}$, Z. Sosin ${ }^{4}$, L. Stuhl ${ }^{27}$, W. Trautmann ${ }^{10}$, A. Trifirò ${ }^{7,8}$, M. Trimarchi ${ }^{7,8}$, M.B. Tsang ${ }^{16}$, G. Verde ${ }^{1,34}$, M. Veselsky ${ }^{35}$, M. Vigilante ${ }^{31,32}$, Yongjia Wang ${ }^{30}$, A. Wieloch ${ }^{4}$, P. Wigg ${ }^{18}$, J. Winkelbauer ${ }^{16}$, H.H. Wolter ${ }^{36}$, P. Wu ${ }^{18}$, S. Yennello ${ }^{15}$, P. Zambon ${ }^{22,23}$, L. Zetta ${ }^{22,24}$, and M. Zoric ${ }^{12}$

\footnotetext{
${ }^{1}$ INFN-Sezione di Catania, Catania, Italy

${ }^{2}$ INFN-Laboratori Nazionali del Sud, Catania, Italy

${ }^{3}$ Universidad Nacional Autònoma de Mèxico, Mexico

${ }^{4}$ Marian Smoluchowski Institute of Physics, Jagiellonian University, Kraków, Poland

${ }^{5}$ KACST Riyadh, Riyadh, Saudi Arabia

${ }^{6}$ King Saud University, Riyadh, Saudi Arabia

${ }^{7}$ INFN-Gruppo Collegato di Messina, Messina, Italy

${ }^{8}$ Dip. MIFT Università di Messina, Messina, Italy

${ }^{9}$ Technische Universität, Darmstadt, Germany

${ }^{10} \mathrm{GSI}$ Helmholtzzentrum, Darmstadt, Germany

${ }^{11}$ University of Santiago de Compostela, Santiago de Compostela, Spain

${ }^{12}$ Ruder Bošković Institute, Zagreb, Croatia

${ }^{13}$ GANIL, CEA and IN2P3-CNRS, Caen, France

${ }^{14} \mathrm{H}$. Niewodniczański Institute of Nuclear Physics, Kraków, Poland

${ }^{15}$ Texas A\&M University, College Station, USA

${ }^{16}$ NSCL Michigan State University, East Lansing, USA

${ }^{17}$ Western Michigan University, Kalamazoo, USA

${ }^{18}$ University of Liverpool, Physics Department, Liverpool, United Kingdom

${ }^{19}$ IFIN-HH, Măgurele-Bucharest, Romania

${ }^{20}$ Dip. Fisica e Astronomia, Università di Catania, Catania, Italy

${ }^{21}$ Western Michigan University, Kalamazoo, USA

${ }^{22}$ INFN-Sezione di Milano, Milano, Italy
}

\footnotetext{
ae-mail: defilippo@ct.infn.it
} 


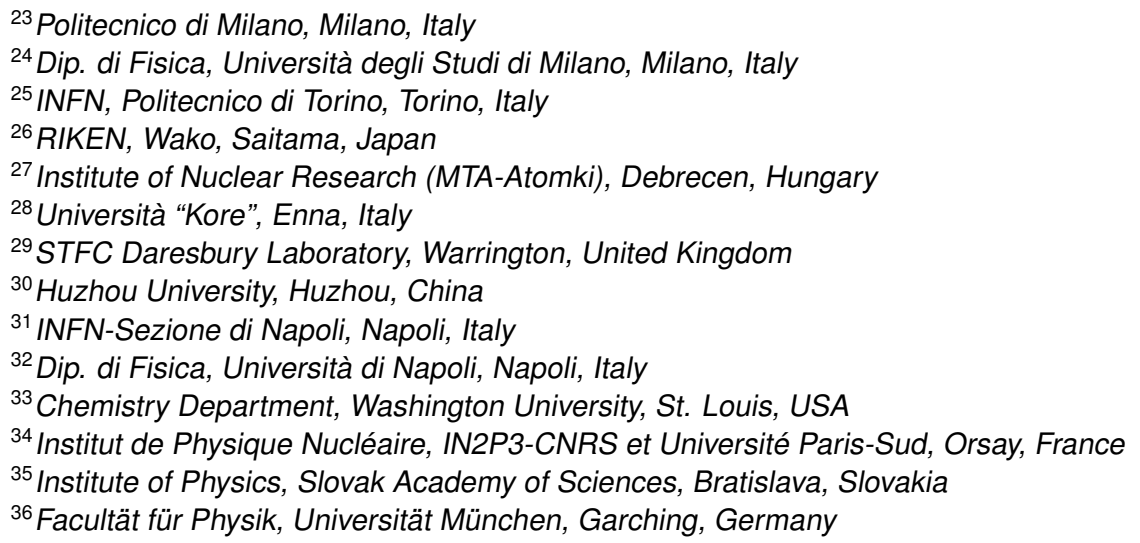

\begin{abstract}
The ASY-EOS experiment at GSI laboratory measured the direct and elliptic flow of neutrons and light charged particles in the reaction ${ }^{197} \mathrm{Au}+{ }^{197} \mathrm{Au}$ at $400 \mathrm{~A} \mathrm{MeV}$ incident energy. The ratio of elliptic flow of neutrons with respect to that of the light charged particles was used as main experimental observable to probe the density dependence of the symmetry energy term of the nuclear equation of state. Results, obtained by comparison of the experimental data with the UrQMD model predictions, strongly support a moderately soft to linear density dependence of the symmetry energy at suprasaturation densities below $2 \rho_{0}$.
\end{abstract}

\title{
1 Introduction
}

The study of the equation of state $\operatorname{EOS}(\rho, T, \beta)$ in neutron rich matter, where $\beta=\left(\rho_{n}-\rho_{p}\right) / \rho$ is the isospin asymmetry as a function of neutrons, protons and nuclear matter density respectively, provides fundamental understanding in many aspects of nuclear physics and astrophysics phenomena [1]. For example, models of the neutron stars properties require as a main input the EOS as a function of a wide range of densities and asymmetry [2]. The main source of uncertainty in describing the effective interaction in the medium is the symmetry energy component of the EOS, which is the difference between the energy per nucleon of neutron matter and the one for symmetric matter. Inconsistencies are particularly evident in the high density matter region above the saturation density $\left(\rho>\rho_{0}\right)$, where diverging results from theoretical predictions are combined with a lack of experimental data [3]. Sources of discrepancies in theoretical calculations are mainly due to uncertainties in nuclear many-body theories, extrapolations of two and three body forces to higher densities and the short range nucleon-nucleon correlations $[4,5]$. In heavy ion collisions transient densities up to 2 or 3 times the saturation density can be reached in a short timescale $(\approx 20 \mathrm{fm} / \mathrm{c})$, during the dynamics of the collision (compression phase), in the incident energy around $\approx 1 A \mathrm{GeV}$ [6]. Therefore, heavy ion collisions provide unique experimental tools to explore densities larger than the nuclear saturation one in controlled laboratory conditions. The largest set of data available around these energies, mainly on pions and light charged particles, come from the FOPI collaboration [7]. In particular, the FOPI data on $\pi^{-} / \pi^{+}$ratio has been used to probe the symmetry term of EOS at suprasaturation density, with contradicting results in the model description of data [8,9]. The ASY-EOS experiment, conducted in GSI laboratory (S394 experiment), uses the flow observable to constrain the nuclear EOS. Flow parameters were already studied in the past for the ${ }^{197} \mathrm{Au}+{ }^{197} \mathrm{Au}$ at $400 \mathrm{~A} \mathrm{MeV}$ by using the FOPI detector coupled with the LAND neutron array [10]. A recent re-analysis of these data, despite their low 
statistical precision, showed that the ratio of the elliptic flow parameters of neutrons with respect to hydrogen isotopes was an observable particularly suitable to determine the strength of the symmetry energy by comparison of the experimental data with the results of the UrQMD transport model [11] or the Tübingen QMD model [12].

The ASY-EOS experiment has been specifically conceived in order to constrain the symmetry energy behavior of the EOS at supra-saturation densities by measuring direct and elliptic flows of neutrons and light charged particles in the reactions ${ }^{197} \mathrm{Au}+{ }^{197} \mathrm{Au},{ }^{96} \mathrm{Ru}+{ }^{96} \mathrm{Ru}$ and ${ }^{96} \mathrm{Zr}+{ }^{96} \mathrm{Zr}$ at $400 \mathrm{~A}$ $\mathrm{MeV}$ drastically improving the statistical accuracy of the previous experiments [10]. In the following the main results for the reaction ${ }^{197} \mathrm{Au}+{ }^{197} \mathrm{Au}$ are summarized. Finally, numerical simulations results and perspectives for new experiments, in continuations of the program started by the ASY-EOS collaboration, will be discussed.

\section{Experimental setup}

The basic idea behind this experiment is that the ratio of the neutron-proton elliptic flow can probe the high density component of the symmetry energy reflecting the squeeze-out (emission perpendicular to the reaction plane) of nuclear matter from the compressed high density region interaction zone. This is well shown in Fig. 3 of Ref. [11] where, for the ${ }^{197} \mathrm{Au}+{ }^{197} \mathrm{Au}$ at $400 \mathrm{~A} \mathrm{MeV}$ the predictions of the UrQMD model are presented for the elliptic flow $\left(v_{2}\right)$ of neutrons, protons and hydrogen yields for two choices of the stiffness of density dependence of the symmetry energy, described by the powerlaw coefficient $\gamma: E_{\text {sym }}(\rho)=E_{\text {sym }}^{k i n}+E_{\text {sym }}^{\text {pot }}=12 \cdot\left(\rho / \rho_{0}\right)^{2 / 3}+22 \cdot\left(\rho / \rho_{0}\right)^{\gamma}$. It results a large neutron squeeze-out in the stiff case $(\gamma=1.5)$ with respect to the soft one $(\gamma=0.5)$, while proton and hydrogen flows depend weakly on $\gamma$ variations.

The experimental setup is shown in the inset of Fig. 1. In order to measure the direct and elliptic flows of neutron and charged particles emitted ad mid-rapidity, the LAND detector [13] was placed at $45^{0}$ with respect to the beam direction at about $5 \mathrm{~m}$ from the target. A veto-wall of plastic scintillators in front of LAND allows for the neutrons and charged particles discrimination. Flow measurements

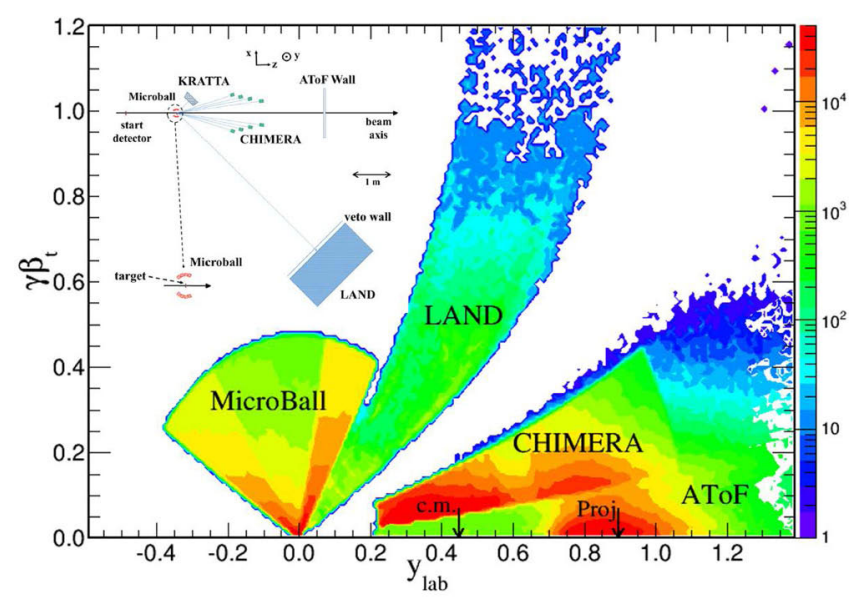

Figure 1. Measured invariant hit distribution in the transverse velocity vs. rapidity plane for charged detected particles for the $\mathrm{Au}+\mathrm{Au}$ reaction. In the top inset, schematic view of the experimental setup. 
require a careful determination of both the reaction plane orientation [14] and the impact parameter. Three devices were used with this goal: i) the Aladin Time-of-Flight wall (ATOF) [15], detecting charged particles up to the polar angle of $7^{0}$; ii) eight rings of the CHIMERA $4 \pi$ detector made by $352 \mathrm{CsI}(\mathrm{Tl})$ scintillators [16] with $2 \pi$ azimuthal coverage around the beam axis and covering polar angles between $7^{0}$ and $20^{\circ}$, were used to detect light charged particles from mid-rapidity emissions; iii) four rings of 50 thin $\mathrm{CsI}(\mathrm{Tl})$ of the $\mu$-ball array [17] surrounding the target at polar angles between $60^{\circ}$ and $147^{\circ}$. Finally, opposite of LAND the KRATTA array [18] permits flow measurements of isotopically resolved light charged particles. Fig. 1 shows the kinematic coverage achieved with this detectors system. The kinematic regime of participant and spectators emissions are clearly evidenced in particular in the CHIMERA and ATOF devices.

\section{Results}

We briefly discuss in this section only the most relevant results of the ASY-EOS experiment. Details of the data analysis can be found in Ref. [19]. Flow parameters for neutrons and light charged particles are constructed looking at their azimuthal distributions with respect to the reference reaction plane. The latter was determined event-by-event by using the CHIMERA and ATOF detectors and the $\mu$ ball, the latter device being mainly used to reject background reactions due to non-target collisions. Flow observables were determined by fitting the azimuthal particle distributions with the Fourier expansion: $f(\Delta \phi) \propto 1+2 \cdot v_{1} \cos (\Delta \phi)+2 \cdot v_{2} \cos (2 \Delta \phi)$ [20], with $v_{1}$ and $v_{2}$ being the direct and elliptic flow coefficients respectively and $\Delta \phi$ the azimuthal angle with respect to the reaction plane. Underestimation of the $v_{1}$ and $v_{2}$ parameters due to fluctuations in the experimental reaction plane determination were corrected using the "sub-events" method of Ollitrault [21].

The experimental ratio (full squares) between $v_{2}^{n} / v_{2}^{c h}$, where $v_{2}^{n}$ and $v_{2}^{c h}$ are respectively the elliptic flows of neutrons and light charged particles (mainly hydrogen isotopes), is shown if Fig. 2 as

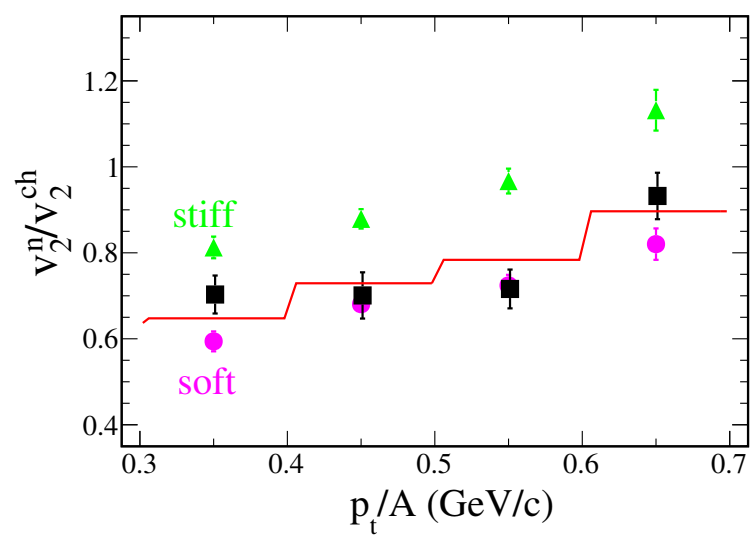

Figure 2. Elliptic flow ratio of neutrons with respect light charged particles for $b<7.5$ fm as a function of the transverse momentum $p_{t} / A$. Black squares are experimental data. Triangles and circles are UrQMD predictions respectively for a stiff and soft power-law exponent $\gamma$ of the symmetry energy potential. 
a function of the transverse momentum $p_{t} / A$ for moderately central (impact parameter $b<7.5 \mathrm{fm}$ ) ${ }^{197} \mathrm{Au}+{ }^{197} \mathrm{Au}$ collisions. The green triangles and red circles represent the UrQMD [22] predictions for stiff $(\gamma=1.5)$ and soft $(\gamma=0.5)$ power-law exponents of the symmetry energy potential term, respectively. Calculations show clearly the sensitivity of the elliptic flow to the stiffness of the symmetry energy. There are several cogent reasons for which the flow ratio $v_{2}^{n} / v_{2}^{c h}$ is here considered: i) from experimental point of view the ratio of the flow parameters minimizes systematic effects in the determination of neutrons or charged particle flows as well as uncertainties related to detectors efficiencies; ii) predictions for the $v_{2}$ flow parameters in the UrQMD model are strongly dependent on the choice made for the momentum dependence of the elastic nucleon-nucleon cross section [23]; this dependence almost vanishes when the flows ratio observable is calculated instead. The solid line in Fig. 2 is the result of a linear interpolation between the UrQMD predictions, weighted according to the experimental error for each $p_{t} / A$ bin. This procedure leads to a value of $\gamma=0.75 \pm 0.10$. Further corrections, including also systematic errors [19], give a final result of $\gamma=0.72 \pm 0.19$. This result shows a moderately soft to linear density dependence of the symmetry energy for a density probed up to twice saturation density. In fact, the sensitivity to density of the elliptic flow ratio was studied for the $\mathrm{Au}+\mathrm{Au}$ reaction at $400 \mathrm{~A} \mathrm{MeV}$ by using the Tübingen QMD transport model $[12,19]$ and the IQMD transport model [24] and it was found that it covers all densities between saturation density and twice this value.

The density dependence of $S(\rho)$ can be expanded in a Taylor series with respect to the density: $S(\rho)=S_{0}+\frac{L}{3}\left(\frac{\rho-\rho_{0}}{\rho_{0}}\right)+\frac{K_{S}}{18}\left(\frac{\rho-\rho_{0}}{\rho_{0}}\right)^{2}+.$. where $L$ and $K_{S}$ are the slope and curvature parameters at $\rho_{0}$

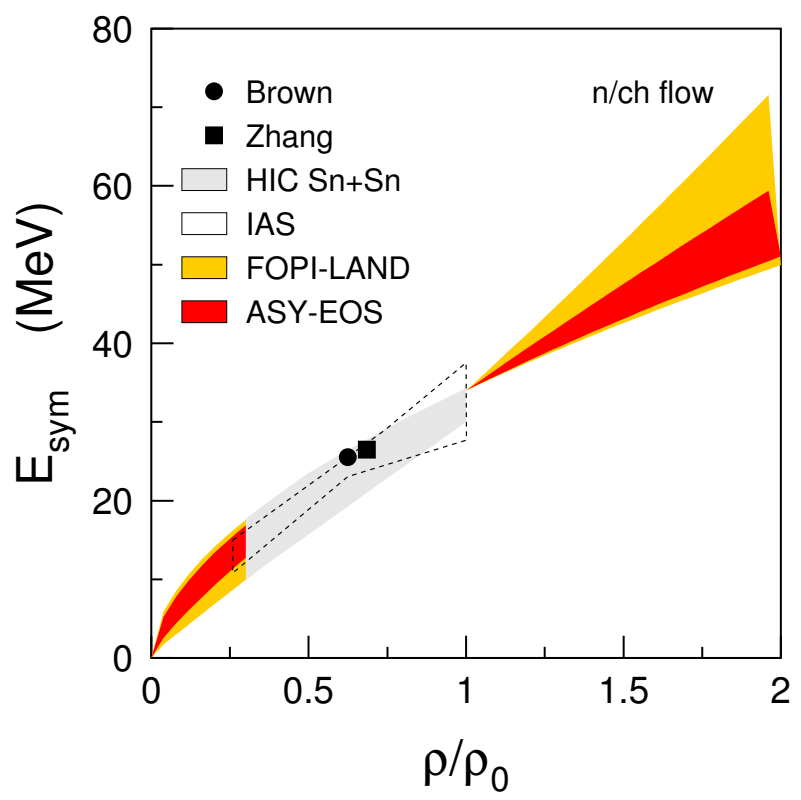

Figure 3. Density dependence of the symmetry energy as deduced from the ASY-EOS data in comparison with the FOPI-LAND data. The grey area (HIC) and the dashed contour (IAS), relative to the low density results, are reported as in Ref. [1] for comparison. 
and $S_{0}$ is the value of symmetry energy at $\rho_{0}$. The slope of the symmetry energy at $\rho_{0}$ is given by $L=3 \rho_{0}\left(\frac{d E_{s y m}(\rho)}{d \rho}\right)_{\rho=\rho_{0}} \approx \frac{3 P_{0}}{\rho_{0}}$ where $P_{0}$ is the pressure of neutron matter at $\rho_{0}$.

Fig. 3 shows the obtained constraint corresponding to the value of $\gamma=0.72 \pm 0.19$ and $L=72 \pm 13$ $\mathrm{MeV}$ as a red band in the $E_{s y m}$ vs. $\rho / \rho_{0}$ plot. The yellow band corresponds to old FOPI-LAND data [11]. This result is in agreement with the constraints on the symmetry energy obtained by using the Tübingen QMD transport model [12] where the isovector parametrization of the symmetry energy has a momentum dependent potential (this ingredient is not present in UrQMD calculations). Anyway these results are in constrast with conclusions from an earlier analysis of FOPI data based on pion $\pi^{-} / \pi^{+}$ratio using the IBUU04 model, giving a super-soft behavior of $E_{\text {sym }}(\rho)$ at supra-saturation densities [8].

\section{Future projects and Conclusions}

There are yet different open problems, questions and uncertainties related to the high density behavior of the symmetry energy [25]. The ASY-EOS experimental results, including the advances in theoretical models they have triggered, are a good starting point to continue this kind of measurements in the future by using improved detection devices and new available radioactive beams at FAIR where systems with larger asymmetries could be studied.

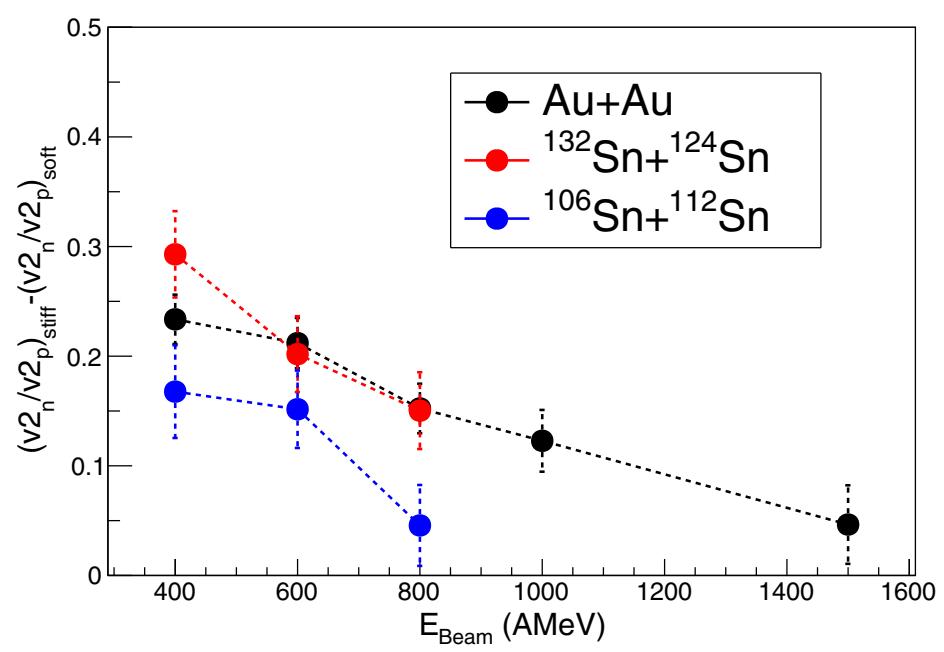

Figure 4. UrQMD calculations showing the model sensitivity to the difference of the neutron/proton $v_{2}$ ratio $\left(v_{2}^{n} / v_{2}^{p}\right)_{s t i f f}-\left(v_{2}^{n} / v_{2}^{p}\right)_{s o f t}$ as a function of the incident beam energies for the indicated systems.

A first point that can be addressed is to explore the sensitivity of the neutron/protons flows ratio observable at higher densities increasing the beam incident energies in the region between $400 \mathrm{~A}$ $\mathrm{MeV}$ and $1500 A \mathrm{MeV}$. Calculations show that higher central densities are reached, with the increase of the collision energies, up to values of $3 \rho / \rho_{0}$ at $1 A \mathrm{GeV}$ [26]. Fig. 4 shows results of UrQMD calculations in which the sensitivity of the elliptic flow ratio of neutrons with respect to protons to the symmetry energy parametrization is reported as a function of beam incident energies. Sensitivity

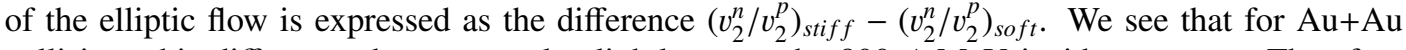
collisions this difference decreases only slightly up to the $800 \mathrm{~A} \mathrm{MeV}$ incident energy. Therefore 
future experiments can significantly explore higher-density regions by using the elliptic flow ratio of neutrons and protons as main observable for the stiffness of the symmetry energy. Because in this new experiment a good identification of neutrons and isotopically separated hydrogen with high resolution is a fundamental task, it is envisaged to use the NeuLAND [27] detector, with improved detector efficiency with respect to LAND, that is part of the $R^{3} B$ project for FAIR at GSI. This could be a fundamental step towards more complex experiments at FAIR with stable and radioactive beams in which several relevant observables sensitive to the stiffness of the symmetry energy should be studied simultaneously, like flows of isospin pairs (like $\mathrm{n} / \mathrm{p}, \mathrm{t} /{ }^{3} \mathrm{He}$ ), ratios of particles production (like $\pi^{-} / \pi^{+}$, $K^{+} / K^{0}$ ratios) and new observable, as recently proposed [9], like high energy photons.

In conclusion, we have shown results from the measurement of the elliptic flows of neutrons and light charged particles in the reaction ${ }^{197} \mathrm{Au}+{ }^{197} \mathrm{Au}$ at $400 \mathrm{~A} \mathrm{MeV}$. From this experiment a stringent constraint for the symmetry energy at supra-saturation density has been deduced, Also, contributions have been given in the direction to improve the understanding of the models by careful comparison of experimental data with transport codes. Indeed we have shown that results of this experiment are an important starting point for future experiments and opportunities offered by new facilities like FAIR.

\section{References}

[1] C.J. Horowitz et al., J. of Phys. G: Nucl. Part. Phys. 41, 093001 (2014).

[2] A.W. Steiner and S. Gandolfi, Phys. Rev Lett. 108, 081102 (2012).

[3] W. Trautmann and H.H. Wolter, Int. J. Mod. Phys. E21, 1220003 (2012).

[4] Wei Zuo, I. Bombaci and U. Lombardo, Eur. Phys. J. A50, 12 (2014).

[5] O. Hen et al., Phys. Rev. C91, 025803 (2015).

[6] Bao-An Li et al., Nucl. Phys. A708, 365 (2002).

[7] W. Reisdorf et al., Nucl. Phys. A781, 459 (2007); ibid. Nucl. Phys. A876, 1 (2012);

[8] Z. Xiao et al. Phys. Rev. Lett. 102, 062502 (2009).

[9] Z. Xiao et al., Eur. Phys. J. A50, 37 (2014).

[10] Y. Leifels et al., Phys. Rev. Lett. 71, 963 (1993).

[11] P. Russotto et al., Phys. Lett. B697, 471 (2011).

[12] M.D. Cozma et al., Phys. Rev. C88, 044912 (2013).

[13] T. Blaich et al. Nucl. Instr. Meth. 314, 136 (1992).

[14] J.-Y. Ollitrault, Nucl. Phys. A638 195c (1998).

[15] A. Schüttauf et al., Nucl. Phys. A607 457 (1996).

[16] A. Pagano et al., Nucl. Phys. A734 504 (2004).

[17] D.G. Sarantites et al. Nucl. Instr. Meth. 381, 418 (1996).

[18] J. Łukasik et al. Nucl. Instr. Meth. A709, 120 (2013).

[19] P. Russotto et al., Phys. Rev. C94, 034608 (2016).

[20] A. Andronic et al., Eur. Phys. J. A30, 31 (2006).

[21] J.-Y. Ollitrault, arXiv:nucl-ex/9711003 (1997).

[22] Q. Li et al., J. Phys. G31, 1359 (2005).

[23] Q. Li et al., Phys. Rev. C83, 044617 (2011).

[24] A. Le Fèvre etal., Nucl. Phys. A945, 112 (2016).

[25] Bao-An Li, arXiv:nucl-th/1610.00549 (2016).

[26] Jun Xu et al., Phys. Rev. C87, 067601 (2013).

[27] NeuLAND Technical Design Report (2011). 